Relations industrielles

Industrial Relations

\title{
The Role of Trade Unions in Developing Societies, by Everett M. Kassalow and Ikanig Damachi (Eds), Geneva, International Institue for Labour Studies, 1978, 183 pp.
}

\section{Gérard Dion}

Volume 34, numéro 1, 1979

URI : https://id.erudit.org/iderudit/028956ar

DOI : https://doi.org/10.7202/028956ar

Aller au sommaire du numéro

Éditeur(s)

Département des relations industrielles de l'Université Laval

ISSN

0034-379X (imprimé)

1703-8138 (numérique)

Découvrir la revue

Citer ce compte rendu

Dion, G. (1979). Compte rendu de [The Role of Trade Unions in Developing

Societies, by Everett M. Kassalow and Ikanig Damachi (Eds), Geneva,

International Institue for Labour Studies, 1978, 183 pp.] Relations industrielles /

Industrial Relations, 34(1), 210-211. https://doi.org/10.7202/028956ar

Tous droits réservés @ C Département des relations industrielles de l'Universite Laval, 1979
Ce document est protégé par la loi sur le droit d'auteur. L'utilisation des services d’Érudit (y compris la reproduction) est assujettie à sa politique d'utilisation que vous pouvez consulter en ligne.

https://apropos.erudit.org/fr/usagers/politique-dutilisation/ 
lité volontaire du salarié et de son coût, est elle-même limitée.

Il faudra sans doute ré-introduire certains éléments de l'analyse «institutionnaliste" tels la relation entre formation en cours d'emploi et processus de socialisation dans le milieu de travail, instabilité d'emploi et organisation (formelle ou informelle) du «job temtory», salaires et structures sociales de rémunération.

\section{Jacques MERCIER}

Université Laval

Le fondateur du syndicalisme catholique au Québec, Mgr Lapointe, par Robert Parisée, Montréal, Les Presses de l'Université du Québec, 1978, 80 pp.

Mgr Eugène Lapointe est certainement une grande personnalité qui a imprimé sa marque sur le développement de la pensée et de l'action sociales de la région du Saguenay au début du siècle. Son zèle, son dévouement pour l'amélioration de la condition des classes laborieuses ont inspiré beaucoup d'autres apôtres sociaux à œuvrer dans ce domaine. Il est devenu un personnage légendaire.

L'auteur nous avertit que «son ouvrage a pour objectif d'exposer systématiquement la pensée et l'action de $\mathrm{Mgr}$ Eugène Lapointe dans le domaine syndical exclusivement" et qu'il repose, pour l'essentiel sur un travail de recherche effectué aux archives de la Société historique du Saguenay.

Six très brefs chapitres constituent l'ouvrage. Ceux qui ont connu $\mathrm{Mgr}$ Lapointe resteront sur leur appétit. Quant aux autres, je crains qu'ils ne pourront jamais s'expliquer pourquoi Mgr Lapointe a pu jouir d'une telle réputation.

Ce n'est pas une étude fouillée ni systématique de la pensée du personnage. On n'y apprend pas beaucoup plus que ce que l'on trouve dans l'article de Michel Têtu publié dans Relations industrielles en 1962.

Faute d'être replacées dans leur contexte idéologique et historique, les conceptions et les positions de Mgr Lapointe peuvent difficilement être comprises par le lecteur contemporain et risquent plutôt de discréditer le personnage et son auvre.

Répéter aujourd'hui que Mgr Lapointe a été le fondateur du syndicalisme catholique au Québec, ce n'est pas simplement une exagération mais c'est une erreur. En effet, malgré les bonnes intentions et le dévouement de son fondateur, la Fédération ouvrière de Chicoutimi de 1907 n'était pas à proprement parler du syndicalisme, mais simplement une société mutuelle dont l'efficacité a été douteuse et dont la durée a été éphémère. Constater cette vérité, ce n'est pas une injure à la mémoire de $\mathrm{Mgr}$ Lapointe; ce n'est pas non plus nier le travail qu'il a accompli, car celui-ci donnera ses fruits plus tard et la région du Saguenay deviendra un des foyers les plus actifs du syndicalisme catholique au Québec.

Il aurait été intéressant d"expliquer comment $\mathrm{Mgr}$ Lapointe en était venu à sa conception d'action ouvrière qu'il considérait comme du syndicalisme, où il avait pris ses sources d'inspiration, à quelle école de pensée il se rattachait, pourquoi il avait choisi cette école plutôt qu'une autre, quels problèmes cela lui a causé au Québec avec d'autres penseurs sociaux instigateurs du syndicalisme catholique qui appartenaient à une autre école, comme l'abbé Maxime Fortin et Alfred Charpentier, etc. Mais pour en arriver là, l'auteur aurait du pousser ses recherches au delà des archives personnelles de $\mathrm{Mgr}$ Lapointe qui, tout intéressantes qu'elles soient, m'apparaissent assez limitées.

Cet ouvrage relève beaucoup plus de la littérature édifiante que de l'histoire. Mgr Lapointe aurait mérité mieux que cela.

Gérard DION

Université Laval

The Role of Trade Unions in Developing Societies, by Everett M. Kassalow and Ikanig Damachi (Eds), Geneva, International Institute for Labour Studies, $1978,183 \mathrm{pp}$.

Les études sur le syndicalisme dans les pays en voie de développement ont 
grandement contribué à enrichir les connaissances sur cette institution et à dissocier certaines variables qu'on lui croyait inhérentes à partir seulement de l'observation faite dans les pays de longue tradition industrielle.

À la fin des années ' 50 et au début des années ' 60 , Walter Galenson, avec une équipe de collaborateurs, avait déjà effectué des études comparatives dans un certain nombre de pays et s'était permis d'élaborer certaines prévisions sur la nature, la place, le rôle et l'action des syndicats. Vingt ans ont passé, l'Institut international d'études sociales, grâce encore à un groupe de collaborateurs, présente dans un même ouvrage une série de monographies ne portant pas sur les mêmes pays (sauf le Chili) mais avec la même méthode. Ce sont le Ghana, Singapore, la Grèce, le Chili, les Philippines, le Kenya et la Corée.

On comprend facilement les difficultés rencontrées pour rassembler une collaboration aussi variée. Et les études ne sont pas toutes récentes. Deux collègues canadiens sont au nombre des collaborateurs: Chris Jecchinis, de l'Université Lakehead pour la Grèce et le regretté
Douglas Muir pour le Kenya dont les lecteurs de Relations industrielles seront heureux de relire le texte déjà paru dans le volume 29 (1974) no. 3, pp. 474-496.

Il serait trop long d'entrer dans les particularités de chacune des études. L'introduction d'Everett M. Kassalow est vraiment remarquable. Avec ses vastes connaissances du problème et l'esprit de synthèse qu'on lui connaît, non seulement il dégage les constantes rencontrées dans toutes les monographies de l'ouvrage, mais il fait une étude comparative avec les observations et les prévisions de Galenson, Sturnthal, Miller et autres qui ont déjà écrit sur le sujet. Sans vouloir minimiser les monographies sans lesquelles Kassalow n'aurait pu faire sa synthèse, cette introduction à elle seule vaut la peine qu'on se procure l'ouvrage. Elle est indispensable à tous ceux qui veulent comprendre le phénomène syndical non seulement dans les pays en voie de développement mais dans les autres pays. C'est une contribution essentielle à l'élaboration de toute théorie du syndicalisme.

Gérard DION

Université Laval

\title{
COLLECTION RELATIONS DU TRAVAIL
}

\author{
Les débrayages massifs de mai 1972 au Québec \\ Gilles LAFLAMME et Réal ALLARD \\ département des relations industrielles \\ Université Laval
}

Un volume $81 / 2 \times 11,50$ pages $-A$ Book $81 / 2 \times 11,50$ pages

\author{
Les Presses de I'Université Laval \\ Cité Universitaire \\ Québec, P.Q., Canada \\ G1K 7R4
}

